\title{
Recomendación de Ramas: Actualizaciones en el diagnóstico y tratamiento de la Hipertensión Arterial en Pediatría. Rama de Nefrología, Sociedad Chilena de Pediatría.
}

\author{
EDDA LAGOMARSINO F. ${ }^{1}$, CARLOS SAIEH A. ${ }^{2}$, MARLENE AGLONY I. ${ }^{3}$ \\ 1. Nefrólogo Pediatra, Profesor Titular, Pontificia Universidad Católica de Chile. \\ 2. Nefrólogo Pediatra, Profesor Agregado, Universidad de Chile. Clínica Las Condes. \\ 3. Nefrólogo Pediatra, Instructor Asociado, Pontificia Universidad Católica de Chile.
}

\begin{abstract}
Up To Date in the diagnosis and treatment of high blood pressure in Pediatrics. Recommendations from the Nephrology Branch of the Chilean Society of Pediatrics

Blood pressure (BP) is a vital sign routinely obtained in adult physical examination. This is not the case in children; therefore, high blood pressure in children is frequently not diagnosed. It should be measured with adequate equipment according to age and height of the child, considering that BP values increase under physiological conditions. Arterial hypertension is defined in percentiles for age, gender and height. Three categories can be established: normal BP, pre-hypertension and hypertension. Clinical studies have determined that the younger the child, the probability of secondary hypertension increases, usually of renal origin. Genetic and metabolic risk factors have been identified intrauterine; this "fetal programming" is related later in life with the onset of high blood pressure. Arterial hypertension evolves without symptoms for long periods of time, making more relevant a complete physical examination that includes BP. The hypertensive patient must be approached by age, clinical history, physical examination and BP values, followed by a laboratory work-up. Complementary studies including BP ambulatory monitoring are being used with increasing frequency in the pediatric population, allowing a big number of BP readings during diary activities of the child. Arterial hypertension treatment in pediatrics begins with the prevention of known risk factors, encouraging a change of lifestyle for the child and his/her family. Drug treatment must be reserved after secondary causes have been corrected and lifestyle modifications did not work out. Pharmacological treatment must be indicated individually, its efficacy monitored and potential adverse effects assessed. Still at an experimental stage, antihypertensive vaccination modifying the renin-angiotensin system is being studied.
\end{abstract}

(Key words: Blood pressure, arterial hypertension, diagnosis, lifestyle, drug therapy).

Rev Chil Pediatr 2008; 79 (1): 63-81

Trabajo recibido el 12 de noviembre de 2007, devuelto para corregir el 17 de diciembre de 2007, segunda versión el 09 de enero de 2008, aceptado para publicación el 16 de enero de 2008.

Correspondencia a:

Edda Lagomarsino F.

E-mail: elagomar@med.puc.cl 


\section{RESUMEN}

La presión arterial (PA), a pesar de ser un signo vital, no se registra habitualmente en pediatría lo que hace que la hipertensión arterial esté sub diagnosticada. El registro de la presión arterial debe hacerse cumpliendo criterios consensuados, con los aparatos adecuados para la edad y talla del niño, ya que los valores de presión arterial aumentan progresivamente en condiciones fisiológicas. La definición de Hipertensión arterial se basa en percentiles para edad, sexo y talla y se distinguen tres etapas: presión normal, pre hipertensión e hipertensión. Mientras menor es el niño es más probable que su hipertensión sea secundaria y tenga origen renal. Se han identificado factores de riesgo genético que aún no podemos prevenir y metabólicos especialmente en la vida intrauterina o "programación fetal" que se relaciona con la presencia de hipertensión arterial posterior. La hipertensión arterial evoluciona asintomática por largos períodos, lo que hace más importante su búsqueda sistemática. El estudio del paciente hipertenso debe orientarse de acuerdo a la edad, los antecedentes anamnésticos, los hallazgos del examen físico y las cifras de presión encontradas para lo que hay estudios de laboratorio sistematizados en fases. El estudio complementario con la monitorización ambulatoria de presión arterial está siendo utilizado frecuentemente en la población pediátrica porque permite tener un gran número de mediciones durante las actividades regulares del niño. El tratamiento de la hipertensión arterial en pediatría comienza con la prevención de los factores de riesgo conocidos propiciando cambios de estilos de vida en él y su familia. El tratamiento farmacológico debe indicarse cuando se han corregido las causas secundarias y/o la modificación de los estilos de vida no han logrado un resultado satisfactorio. El tratamiento farmacológico debe ser individualizado, monitorizando su eficacia y los potenciales efectos secundarios. En etapa experimental está el uso de vacunas antihipertensivas que modifican el sistema renina angiotensina.

(Palabras clave: Presión arterial, hipertensión arterial, diagnóstico, estilos de vida, terapia farmacológica). Rev Chil Pediatr 2008; 79 (1): 63-81

\section{Introducción}

El control de la presión arterial (PA) no se hace habitualmente en la consulta pediátrica, a pesar de tratarse de un signo vital importante. Ello hace que la hipertensión arterial (HTA) esté sub diagnosticada en este grupo etario. Su prevalencia se estima en el $1 \%$ en la población pediátrica ${ }^{1}$.

Actualmente se puede asegurar que la presión elevada tiene sus inicios en la niñez, desde la vida intrauterina, existiendo una tendencia claramente establecida de perpetuación de los niveles de PA en el tiempo, es decir, que aquellos niños con valores de presión altos, cercanos al percentil 90 tendrán en la adolescencia o en la edad adulta más probabilidades de ser hipertensos que los niños con valores en los percentiles más bajos².

Por otro lado, el daño cardiovascular y a otros parénquimas secundarios a la HTA ha hecho plantear cada vez más la importancia de la toma rutinaria de la PA como parte del examen físico de la población infantil, porque además de identificar hipertensos que requieren tratamiento, descubre aquellos niños susceptibles de desarrollar HTA en la edad adulta y por ende, permite utilizar medidas preventivas que eventualmente eviten o retrasen su aparición ${ }^{3}$.

La prevención debe comenzar en la niñez con estilos de vida saludable y control anual de peso, talla y PA desde los tres años de edad ${ }^{4}$.

Hay cada vez más evidencias que sugieren que la HTA en niños es una condición tratable que merece igual preocupación en el diagnóstico y terapia que la HTA del adulto ${ }^{5}$.

Objetivo: Actualizar las recomendaciones en el diagnóstico y manejo de la hipertensión arterial en pediatría.

\section{¿Cómo tomar la presión arterial?}

Existe consenso en que la toma de PA en pediatría debe cumplir con varios criterios que permiten que los resultados sean comparables ${ }^{2-6}$. Lo primero es tener un niño en un ambiente tranquilo, temperado y cómodo, debe explicarse el procedimiento, con un reposo previo de 5 
minutos o más y sin haber ingerido medicamentos. La posición debe ser sentado, con la espalda y los pies bien apoyados. En los lactantes se acepta la posición supina. La fosa ante cubital derecha debe elevarse a nivel del corazón ${ }^{6}$. Uno de los puntos más importantes es elegir correctamente el brazalete a utilizar. La vejiga de goma debe ser la más adecuada para el tamaño del brazo del niño: el ancho $40 \%$ de la circunferencia del brazo en el punto medio, entre el olecranon y el acromion (2/3 del brazo) y el largo 80 a $100 \%$ de la circunferencia del brazo. Existen en el mercado brazaletes de varios tamaños por lo que se debe considerar las siguientes medidas de acuerdo a la edad del niño, en menores de 1 año $=5 \mathrm{~cm}$, de 1 a 3 años $=$ 5 a $8 \mathrm{~cm}$. De 4 a 8 años $=9$ a $10 \mathrm{~cm}$ y de 9 a 15 años $=10$ a $12 \mathrm{~cm}$.

El registro de PA debe realizarse con el instrumento adecuado, siendo los más utilizados: el esfigmomanómetro de mercurio: técnica usada de referencia. Se ubica el pulso cubital y se insufla suavemente a 20 ó $30 \mathrm{~mm}$ de $\mathrm{Hg}$ hasta el momento en que desaparece el pulso y luego se hace lentamente la deflación. Se registra el menor valor obtenido luego de dos o tres determinaciones. Consignar los pulsos. Si el paciente tiene valores altos, tomar la $\mathrm{PA}$ en tres oportunidades diferentes y los pulsos en las cuatro extremidades, acostado o sentado y de pie. El primer ruido de Korotkoff corresponde a la PA sistólica y el $5^{\circ}$ a la PA diastólica. En ocasiones el $5^{\circ}$ ruido puede escucharse hasta 0 . En este caso debe repetirse la toma de PA y ser cuidadosos en apoyar más suavemente el estetoscopio en la fosa cubital. En caso de persistir auscultándose el último ruido hasta 0 , debe considerarse el cuarto ruido como presión diastólica ${ }^{4-6}$. El Oscilómetro (Dynamap): método ampliamente difundido, especialmente en los pacientes hospitalizados, ya que este método elimina errores del operador, permite múltiples mediciones, puede programarse y entrega PA sistólica, diastólica, media y pulsos. Se estima que puede subestimar la PA sistólica en 6 mmhg y la PA diastólica sobreestimarla en 3 mmh. Cada vez que nos encontremos frente a una toma de PA por sobre el percentil 90 para edad, sexo y talla se debe corroborar con método de auscultación ${ }^{4-6}$.

\section{¿Cuándo tomar la Presión Arterial?}

Todo niño recién nacido debe tener una toma de PA a las 48 horas de vida y este dato debe quedar registrado al igual que el resto de los signos vitales, su peso y talla de nacimiento y el peso de la placenta ${ }^{7}$.

Se debe tomar la PA a todo niño mayor de 3 años de edad una vez al año en su control sano y a los menores de esta edad que tienen factores de riesgo conocidos de HTA como la prematurez, bajo peso de nacimiento, complicación neonatal (uso de catéter umbilical) el antecedente familiar de HTA, cardiopatía congénita (reparada o no reparada), infecciones del tracto urinario recurrentes, hematuria y/o proteinuria, enfermedad renal conocida o malformaciones urológica, historia familiar de enfermedad renal congénita, trasplante de órgano sólido o de médula ósea, paciente oncológico, tratamiento con drogas que puedan elevar la PA, enfermedades sistémicas asociadas con HTA: neurofibromatosis, esclerosis tuberosa y la evidencia de aumento de presión intracraneal.

\section{Definiciones}

Los valores de PA aumentan progresivamente en condiciones fisiológicas y varían según la edad. Durante el primer mes de vida la PA sistólica aumenta de forma rápida, enlenteciéndose posteriormente hasta los 5 años. Entre esta edad y el inicio de la pubertad, la PA sistólica aumenta a un ritmo de 1-2 mmhg y la PA diastólica de 0,5-1 mmhg por año, con mínimas diferencias entre niños y niñas. La edad comprendida entre 13 y 18 años se caracteriza por un notable incremento de los valores de PA, siendo más evidente en los niños que en las niñas ${ }^{2-8}$. Estos cambios se deben al crecimiento y desarrollo corporal, por lo que los valores de normalidad deben tener en cuenta, además de la edad y el sexo, el tamaño corporal. Dado que la talla es, de los indicadores de maduración, el que mejor se correlaciona con la edad esquelética, debe relacionarse los valores de PA, además de la edad y el sexo, con la talla ${ }^{9}$.

Existen tablas estandarizadas de presión sanguínea que permiten rotular a un paciente de hipertenso. Todas las definiciones se realizan 
en base a percentiles para edad, sexo y talla. En la IV publicación sobre diagnóstico y manejo de hipertensión en niños y adolescentes se redefinen y clasifican los estados hipertensivos en tres categorías: normotensión cuando la PA sistólica y diastólica son menores del percentil 90, pre hipertensión, categoría en la cual la PA sistólica y/o diastólica es mayor o igual al percentil 90, pero bajo el percentil 95; o cuando un adolescente tiene una PA mayor o igual a 120/80 mmhg e hipertensión arterial: PA sistólica y/o diastólica mayores o igual al percentil 95, en por lo menos 3 mediciones. En esta categoría se describe estadio 1: PA sistólica y/o PA diastólica entre percentil 95 y percentil 99 más 5 mmhg. Y Estadio 2: PA sistólica y/o diastólica mayor a percentil 99 más 5 mmhg $^{7}$ (tablas 1 y 2).

Lo anterior permite programar el seguimiento de estos niños. Los con PA normal se evaluarán en control sano anual, los pre hipertensos se reevaluarán cada 6 meses y los hipertensos en estadio 1 deben reevaluarse en 1 a 2 semanas o antes si el paciente presenta síntomas. $\mathrm{Si}$ la PA permanece elevada en 2 ocasiones más se debe iniciar evaluación o referir a un especialista dentro del período de 1 mes y en estadio 2 iniciar evaluación o referir al especialista dentro de 1 semana o inmediatamente si el paciente está sintomático.

Es importante reconocer la hipertensión de delantal blanco, situación en la cual el paciente se encuentra con PA por sobre el percentil 95 en la consulta médica, siendo normotenso fuera del ambiente clínico. Estos niños se benefician realizando una monitorización ambulatoria de la PA (MAPA).

Tabla 1a. Mujeres. PAS (mmHg)

\begin{tabular}{|c|c|c|c|c|c|c|c|c|c|}
\hline $\begin{array}{l}\text { Edad } \\
\text { (años) }\end{array}$ & $\begin{array}{l}\text { Percentil de Talla } \\
\text { (NHANES 2000) }\end{array}$ & & 5 & 10 & 25 & 50 & 75 & 90 & 95 \\
\hline 2 & $\begin{array}{l}\text { Estadio } 2 \text { HTA } \\
\text { Estadio1 HTA } \\
\text { Prehipertensión } \\
\text { Normotensión }\end{array}$ & $\mathrm{cms}$ & $\begin{array}{l}79,6 \\
114 \\
102 \\
98 \\
85\end{array}$ & $\begin{array}{l}80,9 \\
115 \\
103 \\
99 \\
85\end{array}$ & $\begin{array}{l}83,0 \\
116 \\
104 \\
100 \\
87\end{array}$ & $\begin{array}{l}85,4 \\
117 \\
105 \\
101 \\
88\end{array}$ & $\begin{array}{l}87,7 \\
119 \\
107 \\
103 \\
89\end{array}$ & $\begin{array}{l}89,9 \\
120 \\
108 \\
104 \\
91\end{array}$ & $\begin{array}{l}91,1 \\
121 \\
109 \\
105 \\
91\end{array}$ \\
\hline 3 & $\begin{array}{l}\text { Estadio } 2 \text { HTA } \\
\text { Estadio1 HTA } \\
\text { Prehipertensión } \\
\text { Normotensión }\end{array}$ & $\mathrm{cms}$ & $\begin{array}{l}87,8 \\
116 \\
104 \\
100 \\
86\end{array}$ & $\begin{array}{l}89,2 \\
116 \\
104 \\
100 \\
87\end{array}$ & $\begin{array}{l}91,6 \\
118 \\
105 \\
102 \\
88\end{array}$ & $\begin{array}{l}94,2 \\
119 \\
107 \\
103 \\
89\end{array}$ & $\begin{array}{l}96,9 \\
120 \\
108 \\
104 \\
91\end{array}$ & $\begin{array}{l}99,3 \\
121 \\
109 \\
106 \\
92\end{array}$ & $\begin{array}{l}100,8 \\
122 \\
110 \\
106 \\
93\end{array}$ \\
\hline 4 & $\begin{array}{l}\text { Estadio } 2 \text { HTA } \\
\text { Estadio1 HTA } \\
\text { Prehipertensión } \\
\text { Normotensión }\end{array}$ & $\mathrm{cms}$ & $\begin{array}{l}94,0 \\
117 \\
105 \\
101 \\
88\end{array}$ & $\begin{array}{l}95,6 \\
118 \\
106 \\
102 \\
88\end{array}$ & $\begin{array}{l}98,1 \\
119 \\
107 \\
103 \\
90\end{array}$ & $\begin{array}{l}101,0 \\
120 \\
108 \\
104 \\
91\end{array}$ & $\begin{array}{l}104,0 \\
122 \\
110 \\
106 \\
92\end{array}$ & $\begin{array}{l}106,8 \\
123 \\
111 \\
107 \\
94\end{array}$ & $\begin{array}{l}108,4 \\
124 \\
112 \\
108 \\
94\end{array}$ \\
\hline 5 & $\begin{array}{l}\text { Estadio } 2 \text { HTA } \\
\text { Estadio1 HTA } \\
\text { Prehipertensión } \\
\text { Normotensión }\end{array}$ & $\mathrm{cms}$ & $\begin{array}{l}100,4 \\
119 \\
107 \\
103 \\
89\end{array}$ & $\begin{array}{l}102,0 \\
119 \\
107 \\
103 \\
90\end{array}$ & $\begin{array}{l}104,8 \\
121 \\
108 \\
105 \\
91\end{array}$ & $\begin{array}{l}108,0 \\
122 \\
110 \\
106 \\
93\end{array}$ & $\begin{array}{l}111,2 \\
123 \\
111 \\
107 \\
94\end{array}$ & $\begin{array}{l}114,3 \\
125 \\
112 \\
109 \\
95\end{array}$ & $\begin{array}{l}116,1 \\
125 \\
113 \\
109 \\
96\end{array}$ \\
\hline 6 & $\begin{array}{l}\text { Estadio } 2 \text { HTA } \\
\text { Estadio1 HTA } \\
\text { Prehipertensión } \\
\text { Normotensión }\end{array}$ & $\mathrm{cms}$ & $\begin{array}{l}106,9 \\
120 \\
108 \\
104 \\
91\end{array}$ & $\begin{array}{l}108,6 \\
121 \\
109 \\
105 \\
92\end{array}$ & $\begin{array}{l}111,6 \\
122 \\
110 \\
106 \\
93\end{array}$ & $\begin{array}{l}115,0 \\
124 \\
111 \\
108 \\
94\end{array}$ & $\begin{array}{l}118,6 \\
125 \\
113 \\
109 \\
96\end{array}$ & $\begin{array}{l}121,9 \\
126 \\
114 \\
110 \\
97\end{array}$ & $\begin{array}{l}123,9 \\
127 \\
115 \\
111 \\
98\end{array}$ \\
\hline 7 & $\begin{array}{l}\text { Estadio } 2 \text { HTA } \\
\text { Estadio1 HTA } \\
\text { Prehipertensión } \\
\text { Normotensión }\end{array}$ & $\mathrm{cms}$ & $\begin{array}{l}113,1 \\
122 \\
110 \\
106 \\
93\end{array}$ & $\begin{array}{l}114,9 \\
123 \\
111 \\
107 \\
93\end{array}$ & $\begin{array}{l}118,1 \\
124 \\
112 \\
108 \\
95\end{array}$ & $\begin{array}{l}121,8 \\
125 \\
113 \\
109 \\
96\end{array}$ & $\begin{array}{l}125,6 \\
127 \\
115 \\
111 \\
97\end{array}$ & $\begin{array}{l}129,1 \\
128 \\
116 \\
112 \\
99\end{array}$ & $\begin{array}{l}131,3 \\
129 \\
116 \\
113 \\
99\end{array}$ \\
\hline
\end{tabular}

Normotensión = percentil 50; Pre-Hipertensión = percentil 90 hasta 80 mmHg; Estadio 1 HTA = percentil 95; Estadio 2 HTA = percentil $99+5 \mathrm{mmHg}$. 
Tabla 1a. Mujeres. PAS (mmHg) (continuación)

\begin{tabular}{|c|c|c|c|c|c|c|c|c|c|}
\hline $\begin{array}{l}\text { Edad } \\
\text { (años) }\end{array}$ & $\begin{array}{l}\text { Percentil de Talla } \\
\text { (NHANES 2000) }\end{array}$ & & 5 & 10 & 25 & 50 & 75 & 90 & 95 \\
\hline \multirow[t]{5}{*}{8} & & $\mathrm{cms}$ & 118,5 & 120,5 & 123,9 & 127,8 & 131,9 & 135,6 & 137,9 \\
\hline & Estadio 2 HTA & & 124 & 125 & 126 & 127 & 128 & 130 & 130 \\
\hline & Estadio1 HTA & & 112 & 112 & 114 & 115 & 116 & 118 & 118 \\
\hline & Prehipertensión & & 108 & 109 & 110 & 111 & 113 & 114 & 114 \\
\hline & Normotensión & & 95 & 95 & 96 & 98 & 99 & 100 & 101 \\
\hline \multirow[t]{5}{*}{9} & & $\mathrm{cms}$ & 123,2 & 125,3 & 129,0 & 133,1 & 137,4 & 141,4 & 143,8 \\
\hline & Estadio 2 HTA & & 126 & 126 & 128 & 129 & 130 & 132 & 132 \\
\hline & Estadio1 HTA & & 114 & 114 & 115 & 117 & 118 & 119 & 120 \\
\hline & Prehipertensión & & 110 & 110 & 112 & 113 & 114 & 116 & 116 \\
\hline & Normotensión & & 96 & 97 & 98 & 100 & 101 & 102 & 103 \\
\hline \multirow[t]{5}{*}{10} & & $\mathrm{cms}$ & 127,5 & 129,8 & 133,7 & 138,2 & 142,8 & 147,0 & 149,6 \\
\hline & Estadio 2 HTA & & 128 & 128 & 130 & 131 & 132 & 134 & 134 \\
\hline & Estadio1 HTA & & 116 & 116 & 117 & 119 & 120 & 121 & 122 \\
\hline & Prehipertensión & & 112 & 112 & 114 & 115 & 116 & 118 & 118 \\
\hline & Normotensión & & 98 & 99 & 100 & 102 & 103 & 104 & 105 \\
\hline \multirow[t]{5}{*}{11} & & $\mathrm{cms}$ & 132,4 & 135,0 & 139,4 & 144,3 & 149,2 & 153,7 & 156,4 \\
\hline & Estadio 2 HTA & & 130 & 130 & 131 & 133 & 134 & 135 & 136 \\
\hline & Estadio1 HTA & & 118 & 118 & 119 & 121 & 122 & 123 & 124 \\
\hline & Prehipertensión & & 114 & 114 & 116 & 117 & 118 & 119 & 120 \\
\hline & Normotensión & & 100 & 101 & 102 & 103 & 105 & 106 & 107 \\
\hline \multirow[t]{5}{*}{12} & & $\mathrm{cms}$ & 139,2 & 142,0 & 146,5 & 151,5 & 156,4 & 160,8 & 163,5 \\
\hline & Estadio 2 HTA & & 132 & 132 & 133 & 135 & 136 & 137 & 138 \\
\hline & Estadio1 HTA & & 119 & 120 & 121 & 123 & 124 & 125 & 126 \\
\hline & Prehipertensión & & 116 & 116 & 117 & 119 & 120 & 120 & 120 \\
\hline & Normotensión & & 102 & 103 & 104 & 105 & 107 & 108 & 109 \\
\hline \multirow[t]{5}{*}{13} & & $\mathrm{cms}$ & 145,9 & 148,4 & 152,7 & 157,3 & 162,0 & 166,1 & 168,6 \\
\hline & Estadio 2 HTA & & 133 & 134 & 135 & 137 & 138 & 139 & 140 \\
\hline & Estadio1 HTA & & 121 & 122 & 123 & 124 & 126 & 127 & 128 \\
\hline & Prehipertensión & & 117 & 118 & 119 & 120 & 120 & 120 & 120 \\
\hline & Normotensión & & 104 & 105 & 106 & 107 & 109 & 110 & 110 \\
\hline \multirow[t]{5}{*}{14} & & $\mathrm{cms}$ & 149,7 & 152,1 & 156,0 & 160,5 & 164,9 & 168,9 & 171,3 \\
\hline & Estadio 2 HTA & & 135 & 136 & 137 & 138 & 140 & 141 & 141 \\
\hline & Estadio1 HTA & & 123 & 123 & 125 & 126 & 127 & 129 & 129 \\
\hline & Prehipertensión & & 119 & 120 & 120 & 120 & 120 & 120 & 120 \\
\hline & Normotensión & & 106 & 106 & 107 & 109 & 110 & 111 & 112 \\
\hline \multirow[t]{5}{*}{15} & & $\mathrm{cms}$ & 151,3 & 153,6 & 157,5 & 161,9 & 166,3 & 170,2 & 172,6 \\
\hline & Estadio 2 HTA & & 136 & 137 & 138 & 139 & 141 & 142 & 143 \\
\hline & Estadio1 HTA & & 124 & 125 & 126 & 127 & 129 & 130 & 131 \\
\hline & Prehipertensión & & 120 & 120 & 120 & 120 & 120 & 120 & 120 \\
\hline & Normotensión & & 107 & 108 & 109 & 110 & 111 & 113 & 113 \\
\hline \multirow[t]{5}{*}{16} & & $\mathrm{cms}$ & 151,9 & 154,3 & 158,2 & 162,6 & 166,9 & 170,9 & 173,2 \\
\hline & Estadio 2 HTA & & 137 & 138 & 139 & 140 & 142 & 143 & 144 \\
\hline & Estadio1 HTA & & 125 & 126 & 127 & 128 & 130 & 131 & 132 \\
\hline & Prehipertensión & & 120 & 120 & 120 & 120 & 120 & 120 & 120 \\
\hline & Normotensión & & 108 & 108 & 110 & 111 & 112 & 114 & 114 \\
\hline \multirow[t]{5}{*}{17} & & $\mathrm{cms}$ & 152,3 & 154,6 & 158,6 & 162,9 & 167,3 & 171,2 & 173,6 \\
\hline & Estadio 2 HTA & & 138 & 138 & 139 & 141 & 142 & 143 & 144 \\
\hline & Estadio1 HTA & & 125 & 126 & 127 & 129 & 130 & 131 & 132 \\
\hline & Prehipertensión & & 120 & 120 & 120 & 120 & 120 & 120 & 120 \\
\hline & Normotensión & & 108 & 109 & 110 & 111 & 113 & 114 & 115 \\
\hline
\end{tabular}

Normotensión = percentil 50; Pre-Hipertensión = percentil 90 hasta $80 \mathrm{mmHg}$; Estadio 1 HTA = percentil 95; Estadio 2 HTA = percentil $99+5 \mathrm{mmHg}$. 
Tabla 1b. Hombres. PAS (mmHg)

\begin{tabular}{|c|c|c|c|c|c|c|c|c|c|}
\hline $\begin{array}{l}\text { Edad } \\
\text { (años) }\end{array}$ & $\begin{array}{l}\text { Percentil de Talla } \\
\text { (NHANES 2000) }\end{array}$ & & 5 & 10 & 25 & 50 & 75 & 90 & 95 \\
\hline 2 & $\begin{array}{l}\text { Estadio } 2 \text { HTA } \\
\text { Estadio1 HTA } \\
\text { Prehipertensión } \\
\text { Normotensión }\end{array}$ & $\mathrm{cms}$ & $\begin{array}{l}81,1 \\
114 \\
101 \\
97 \\
84\end{array}$ & $\begin{array}{l}82,4 \\
115 \\
102 \\
99 \\
85\end{array}$ & $\begin{array}{l}84,5 \\
116 \\
104 \\
100 \\
87\end{array}$ & $\begin{array}{l}86,9 \\
118 \\
106 \\
102 \\
88\end{array}$ & $\begin{array}{l}89,2 \\
120 \\
108 \\
104 \\
90\end{array}$ & $\begin{array}{l}91,4 \\
122 \\
109 \\
105 \\
92\end{array}$ & $\begin{array}{l}92,6 \\
122 \\
110 \\
106 \\
92\end{array}$ \\
\hline 3 & $\begin{array}{l}\text { Estadio } 2 \text { HTA } \\
\text { Estadio1 HTA } \\
\text { Prehipertensión } \\
\text { Normotensión }\end{array}$ & $\mathrm{cms}$ & $\begin{array}{l}89,2 \\
116 \\
104 \\
100 \\
86\end{array}$ & $\begin{array}{l}90,5 \\
117 \\
105 \\
101 \\
87\end{array}$ & $\begin{array}{l}92,7 \\
119 \\
107 \\
103 \\
89\end{array}$ & $\begin{array}{l}95,3 \\
121 \\
109 \\
105 \\
91\end{array}$ & $\begin{array}{l}97,9 \\
123 \\
110 \\
107 \\
93\end{array}$ & $\begin{array}{l}100,4 \\
124 \\
112 \\
108 \\
94\end{array}$ & $\begin{array}{l}101,9 \\
125 \\
113 \\
109 \\
95\end{array}$ \\
\hline 4 & $\begin{array}{l}\text { Estadio } 2 \text { HTA } \\
\text { Estadio1 HTA } \\
\text { Prehipertensión } \\
\text { Normotensión }\end{array}$ & $\mathrm{cms}$ & $\begin{array}{l}95,6 \\
118 \\
106 \\
102 \\
88\end{array}$ & $\begin{array}{l}97,1 \\
119 \\
107 \\
103 \\
89\end{array}$ & $\begin{array}{l}99,7 \\
121 \\
109 \\
105 \\
91\end{array}$ & $\begin{array}{l}102,5 \\
123 \\
111 \\
107 \\
93\end{array}$ & $\begin{array}{l}105,4 \\
125 \\
112 \\
109 \\
95\end{array}$ & $\begin{array}{l}108,0 \\
126 \\
114 \\
110 \\
96\end{array}$ & $\begin{array}{l}109,5 \\
127 \\
115 \\
111 \\
97\end{array}$ \\
\hline 5 & $\begin{array}{l}\text { Estadio } 2 \text { HTA } \\
\text { Estadio1 HTA } \\
\text { Prehipertensión } \\
\text { Normotensión }\end{array}$ & $\mathrm{cms}$ & $\begin{array}{l}101,5 \\
120 \\
108 \\
104 \\
90\end{array}$ & $\begin{array}{l}103,2 \\
121 \\
109 \\
105 \\
91\end{array}$ & $\begin{array}{l}106,0 \\
123 \\
110 \\
106 \\
93\end{array}$ & $\begin{array}{l}109,2 \\
125 \\
112 \\
108 \\
95\end{array}$ & $\begin{array}{l}112,3 \\
126 \\
114 \\
110 \\
96\end{array}$ & $\begin{array}{l}115,1 \\
128 \\
115 \\
111 \\
98\end{array}$ & $\begin{array}{l}116,8 \\
128 \\
116 \\
112 \\
98\end{array}$ \\
\hline 6 & $\begin{array}{l}\text { Estadio } 2 \text { HTA } \\
\text { Estadio1 HTA } \\
\text { Prehipertensión } \\
\text { Normotensión }\end{array}$ & $\mathrm{cms}$ & $\begin{array}{l}107,3 \\
121 \\
109 \\
105 \\
91\end{array}$ & $\begin{array}{l}109,2 \\
122 \\
110 \\
106 \\
92\end{array}$ & $\begin{array}{l}112,2 \\
124 \\
112 \\
108 \\
94\end{array}$ & $\begin{array}{l}115,7 \\
126 \\
114 \\
110 \\
96\end{array}$ & $\begin{array}{l}119,1 \\
128 \\
115 \\
111 \\
98\end{array}$ & $\begin{array}{l}122,1 \\
129 \\
117 \\
113 \\
99\end{array}$ & $\begin{array}{l}123,9 \\
130 \\
117 \\
113 \\
100\end{array}$ \\
\hline 7 & $\begin{array}{l}\text { Estadio } 2 \text { HTA } \\
\text { Estadio1 HTA } \\
\text { Prehipertensión } \\
\text { Normotensión }\end{array}$ & $\mathrm{cms}$ & $\begin{array}{l}113,2 \\
122 \\
110 \\
106 \\
92\end{array}$ & $\begin{array}{l}115,1 \\
123 \\
111 \\
107 \\
94\end{array}$ & $\begin{array}{l}118,4 \\
125 \\
113 \\
109 \\
95\end{array}$ & $\begin{array}{l}122,0 \\
127 \\
115 \\
111 \\
97\end{array}$ & $\begin{array}{l}125,7 \\
129 \\
117 \\
113 \\
99\end{array}$ & $\begin{array}{l}129,0 \\
130 \\
118 \\
114 \\
100\end{array}$ & $\begin{array}{l}131,0 \\
131 \\
119 \\
115 \\
101\end{array}$ \\
\hline 8 & $\begin{array}{l}\text { Estadio } 2 \text { HTA } \\
\text { Estadio1 HTA } \\
\text { Prehipertensión } \\
\text { Normotensión }\end{array}$ & $\mathrm{cms}$ & $\begin{array}{l}118,8 \\
124 \\
111 \\
107 \\
94\end{array}$ & $\begin{array}{l}120,8 \\
125 \\
112 \\
109 \\
95\end{array}$ & $\begin{array}{l}124,3 \\
127 \\
114 \\
110 \\
97\end{array}$ & $\begin{array}{l}128,1 \\
128 \\
116 \\
112 \\
99\end{array}$ & $\begin{array}{l}132,1 \\
130 \\
118 \\
114 \\
100\end{array}$ & $\begin{array}{l}135,7 \\
132 \\
119 \\
115 \\
102\end{array}$ & $\begin{array}{l}137,8 \\
132 \\
120 \\
116 \\
102\end{array}$ \\
\hline 9 & $\begin{array}{l}\text { Estadio } 2 \text { HTA } \\
\text { Estadio1 HTA } \\
\text { Prehipertensión } \\
\text { Normotensión }\end{array}$ & $\mathrm{cms}$ & $\begin{array}{l}123,8 \\
125 \\
113 \\
109 \\
95\end{array}$ & $\begin{array}{l}126,0 \\
126 \\
114 \\
110 \\
96\end{array}$ & $\begin{array}{l}129,6 \\
128 \\
116 \\
112 \\
98\end{array}$ & $\begin{array}{l}133,7 \\
131 \\
118 \\
114 \\
100\end{array}$ & $\begin{array}{l}137,9 \\
132 \\
119 \\
115 \\
102\end{array}$ & $\begin{array}{l}141,8 \\
133 \\
121 \\
117 \\
103\end{array}$ & $\begin{array}{l}144,1 \\
134 \\
121 \\
118 \\
104\end{array}$ \\
\hline 10 & $\begin{array}{l}\text { Estadio } 2 \text { HTA } \\
\text { Estadio1 HTA } \\
\text { Prehipertensión } \\
\text { Normotensión }\end{array}$ & $\mathrm{cms}$ & $\begin{array}{l}128,2 \\
127 \\
115 \\
111 \\
97\end{array}$ & $\begin{array}{l}130,5 \\
128 \\
116 \\
112 \\
98\end{array}$ & $\begin{array}{l}134,4 \\
130 \\
117 \\
114 \\
100\end{array}$ & $\begin{array}{l}138,8 \\
132 \\
119 \\
115 \\
102\end{array}$ & $\begin{array}{l}143,3 \\
133 \\
121 \\
117 \\
103\end{array}$ & $\begin{array}{l}147,4 \\
135 \\
122 \\
119 \\
105\end{array}$ & $\begin{array}{l}149,9 \\
135 \\
123 \\
119 \\
106\end{array}$ \\
\hline 11 & $\begin{array}{l}\text { Estadio } 2 \text { HTA } \\
\text { Estadio1 HTA } \\
\text { Prehipertensión } \\
\text { Normotensión }\end{array}$ & $\mathrm{cms}$ & $\begin{array}{l}132,4 \\
129 \\
117 \\
113 \\
99\end{array}$ & $\begin{array}{l}134,9 \\
130 \\
118 \\
114 \\
100\end{array}$ & $\begin{array}{l}139,0 \\
132 \\
119 \\
115 \\
102\end{array}$ & $\begin{array}{l}143,7 \\
134 \\
121 \\
117 \\
104\end{array}$ & $\begin{array}{l}148,5 \\
135 \\
123 \\
119 \\
105\end{array}$ & $\begin{array}{l}152,9 \\
137 \\
124 \\
120 \\
107\end{array}$ & $\begin{array}{l}155,5 \\
137 \\
125 \\
120 \\
107\end{array}$ \\
\hline
\end{tabular}

Normotensión = percentil 50; Pre-Hipertensión = percentil 90 hasta $80 \mathrm{mmHg}$; Estadio 1 HTA = percentil 95; Estadio 2 HTA = percentil $99+5 \mathrm{mmHg}$. 
Tabla 1b. Hombres. PAS (mmHg) (continuación)

\begin{tabular}{|c|c|c|c|c|c|c|c|c|c|}
\hline $\begin{array}{l}\text { Edad } \\
\text { (años) }\end{array}$ & $\begin{array}{l}\text { Percentil de Talla } \\
\text { (NHANES 2000) }\end{array}$ & & 5 & 10 & 25 & 50 & 75 & 90 & 95 \\
\hline \multirow[t]{5}{*}{12} & & $\mathrm{cms}$ & 137,3 & 139,9 & 144,3 & 149,3 & 154,4 & 159,0 & 161,9 \\
\hline & Estadio 2 HTA & & 131 & 132 & 134 & 136 & 138 & 139 & 140 \\
\hline & Estadio1 HTA & & 119 & 120 & 122 & 123 & 125 & 127 & 127 \\
\hline & Prehipertensión & & 115 & 116 & 118 & 120 & 120 & 120 & 120 \\
\hline & Normotensión & & 101 & 102 & 104 & 106 & 108 & 109 & 110 \\
\hline \multirow[t]{5}{*}{13} & & $\mathrm{cms}$ & 143,6 & 146,4 & 151,1 & 156,4 & 161,7 & 166,6 & 169,5 \\
\hline & Estadio 2 HTA & & 133 & 135 & 136 & 138 & 140 & 141 & 142 \\
\hline & Estadio1 HTA & & 121 & 122 & 124 & 126 & 128 & 129 & 130 \\
\hline & Prehipertensión & & 115 & 116 & 118 & 120 & 120 & 120 & 120 \\
\hline & Normotensión & & 104 & 105 & 106 & 108 & 110 & 111 & 112 \\
\hline \multirow[t]{5}{*}{14} & & $\mathrm{cms}$ & 150,5 & 153,6 & 158,7 & 164,1 & 169,5 & 174,2 & 177,0 \\
\hline & Estadio 2 HTA & & 136 & 137 & 139 & 141 & 143 & 144 & 145 \\
\hline & Estadio1 HTA & & 124 & 125 & 127 & 128 & 130 & 132 & 132 \\
\hline & Prehipertensión & & 120 & 120 & 120 & 120 & 120 & 120 & 120 \\
\hline & Normotensión & & 106 & 107 & 109 & 111 & 113 & 114 & 115 \\
\hline \multirow[t]{5}{*}{15} & & $\mathrm{cms}$ & 156,7 & 159,8 & 164,8 & 170,1 & 175,3 & 179,8 & 182,4 \\
\hline & Estadio 2 HTA & & 139 & 140 & 141 & 143 & 145 & 147 & 147 \\
\hline & Estadio1 HTA & & 126 & 127 & 129 & 131 & 133 & 134 & 135 \\
\hline & Prehipertensión & & 120 & 120 & 120 & 120 & 120 & 120 & 120 \\
\hline & Normotensión & & 109 & 110 & 112 & 113 & 115 & 117 & 117 \\
\hline \multirow[t]{5}{*}{16} & & $\mathrm{cms}$ & 160,8 & 163,7 & 168,5 & 173,6 & 178,6 & 182,9 & 185,5 \\
\hline & Estadio 2 HTA & & 141 & 142 & 144 & 146 & 148 & 149 & 150 \\
\hline & Estadio1 HTA & & 129 & 130 & 132 & 134 & 135 & 137 & 137 \\
\hline & Prehipertensión & & 120 & 120 & 120 & 120 & 120 & 120 & 120 \\
\hline & Normotensión & & 111 & 112 & 114 & 116 & 118 & 119 & 120 \\
\hline \multirow[t]{5}{*}{17} & & $\mathrm{cms}$ & 163,1 & 165,8 & 170,4 & 175,3 & 180,2 & 184,5 & 187,0 \\
\hline & Estadio 2 HTA & & 144 & 145 & 146 & 148 & 150 & 151 & 152 \\
\hline & Estadio1 HTA & & 131 & 132 & 134 & 136 & 138 & 139 & 140 \\
\hline & Prehipertensión & & 120 & 120 & 120 & 120 & 120 & 120 & 120 \\
\hline & Normotensión & & 114 & 115 & 116 & 118 & 120 & 120 & 120 \\
\hline
\end{tabular}

Normotensión = percentil 50; Pre-Hipertensión = percentil 90 hasta $80 \mathrm{mmHg}$; Estadio 1 HTA = percentil 95; Estadio 2 HTA = percentil $99+5 \mathrm{mmHg}$.

\section{Etiología}

Cuanto más joven es el niño y más altos son los valores de PA, más posibilidades hay de que la HTA sea secundaria, siendo las causas de origen renal, parenquimatoso o vascular las más frecuentes (70-80\%). La edad de comienzo orienta sobre las causas más frecuentes. En el recién nacido y el lactante difieren de las que presentan los niños mayores y los adolescentes $^{9}$ (tabla 3).

La elevación de la PA sistólica y diastólica, diurna y nocturna orienta a una causa secundaria, mientras que el aumento de la PA sistólica se observa de preferencia en la HTA esencial $^{10}$.

\section{Factores de Riesgo}

Se han identificado factores de riesgo genético y/o metabólico para una futura PA elevada, debiendo éstos ser conocidos y manejados por el pediatra para prevenir la aparición de la HTA o bien retardarla ${ }^{11}$.

Cuando ambos padres son hipertensos, 50\% de los hijos heredará la condición; si uno solo lo es, la cifra cae al $20-30 \%{ }^{12}$. Frente a antecedentes familiares de HTA, se ha relacionado la elevación de la PA con la resistencia a la insulina, ya que $40 \%$ de los pacientes hipertensos tienen hiperinsulinemia (por aumento de la secreción de insulina y baja de la aclaración renal de ella) y porque hay una correlación directamente proporcional entre PA y niveles de insu- 
Tabla 2a. Mujeres. PAD (mmHg)

\begin{tabular}{|c|c|c|c|c|c|c|c|c|c|}
\hline $\begin{array}{l}\text { Edad } \\
\text { (años) }\end{array}$ & $\begin{array}{l}\text { Percentil de Talla } \\
\text { (NHANES 2000) }\end{array}$ & & 5 & 10 & 25 & 50 & 75 & 90 & 95 \\
\hline \multirow[t]{5}{*}{2} & & $\mathrm{cms}$ & 79,6 & 80,9 & 83,0 & 85,4 & 87,7 & 89,9 & 91,1 \\
\hline & Estadio 2 HTA & & 74 & 74 & 75 & 75 & 76 & 77 & 77 \\
\hline & Estadio1 HTA & & 61 & 62 & 62 & 63 & 64 & 65 & 65 \\
\hline & Prehipertensión & & 57 & 58 & 58 & 59 & 60 & 61 & 61 \\
\hline & Normotensión & & 43 & 44 & 44 & 45 & 46 & 46 & 47 \\
\hline \multirow[t]{5}{*}{3} & & $\mathrm{cms}$ & 87,8 & 89,2 & 91,6 & 94,2 & 96,9 & 99,3 & 100,8 \\
\hline & Estadio 2 HTA & & 78 & 78 & 79 & 79 & 80 & 81 & 81 \\
\hline & Estadio1 HTA & & 65 & 66 & 66 & 67 & 68 & 68 & 69 \\
\hline & Prehipertensión & & 61 & 62 & 62 & 63 & 64 & 64 & 65 \\
\hline & Normotensión & & 47 & 48 & 48 & 49 & 50 & 50 & 51 \\
\hline \multirow[t]{5}{*}{4} & & $\mathrm{cms}$ & 94,0 & 95,6 & 98,1 & 101,0 & 104,0 & 106,8 & 108,4 \\
\hline & Estadio 2 HTA & & 81 & 81 & 81 & 82 & 83 & 84 & 84 \\
\hline & Estadio1 HTA & & 68 & 68 & 69 & 70 & 71 & 71 & 72 \\
\hline & Prehipertensión & & 64 & 64 & 65 & 66 & 67 & 67 & 68 \\
\hline & Normotensión & & 50 & 50 & 51 & 52 & 52 & 53 & 54 \\
\hline \multirow[t]{5}{*}{5} & & $\mathrm{cms}$ & 100,4 & 102,0 & 104,8 & 108,0 & 111,2 & 114,3 & 116,1 \\
\hline & Estadio 2 HTA & & 83 & 83 & 84 & 84 & 85 & 86 & 86 \\
\hline & Estadio1 HTA & & 70 & 71 & 71 & 72 & 73 & 73 & 74 \\
\hline & Prehipertensión & & 66 & 67 & 67 & 68 & 69 & 69 & 70 \\
\hline & Normotensión & & 52 & 53 & 53 & 54 & 55 & 55 & 56 \\
\hline \multirow[t]{5}{*}{6} & & $\mathrm{cms}$ & 106,9 & 108,6 & 111,6 & 115,0 & 118,6 & 121,9 & 123,9 \\
\hline & Estadio 2 HTA & & 85 & 85 & 85 & 86 & 87 & 88 & 88 \\
\hline & Estadio1 HTA & & 72 & 72 & 73 & 74 & 74 & 75 & 76 \\
\hline & Prehipertensión & & 68 & 68 & 69 & 70 & 70 & 71 & 72 \\
\hline & Normotensión & & 54 & 54 & 55 & 56 & 56 & 57 & 58 \\
\hline \multirow[t]{5}{*}{7} & & $\mathrm{cms}$ & 113,1 & 114,9 & 118,1 & 121,8 & 125,6 & 129,1 & 131,3 \\
\hline & Estadio 2 HTA & & 86 & 86 & 87 & 87 & 88 & 89 & 89 \\
\hline & Estadio1 HTA & & 73 & 74 & 74 & 75 & 76 & 76 & 77 \\
\hline & Prehipertensión & & 69 & 70 & 70 & 71 & 72 & 72 & 73 \\
\hline & Normotensión & & 55 & 56 & 56 & 57 & 58 & 58 & 59 \\
\hline \multirow[t]{5}{*}{8} & & $\mathrm{cms}$ & 118,5 & 120,5 & 123,9 & 127,8 & 131,9 & 135,6 & 137,9 \\
\hline & Estadio 2 HTA & & 87 & 87 & 88 & 88 & 89 & 90 & 91 \\
\hline & Estadio1 HTA & & 75 & 75 & 75 & 76 & 77 & 78 & 78 \\
\hline & Prehipertensión & & 71 & 71 & 71 & 72 & 73 & 74 & 74 \\
\hline & Normotensión & & 57 & 57 & 57 & 58 & 59 & 60 & 60 \\
\hline \multirow[t]{5}{*}{9} & & $\mathrm{cms}$ & 123,2 & 125,3 & 129,0 & 133,1 & 137,4 & 141,4 & 143,8 \\
\hline & Estadio 2 HTA & & 88 & 88 & 89 & 89 & 90 & 91 & 92 \\
\hline & Estadio1 HTA & & 76 & 76 & 76 & 77 & 78 & 79 & 79 \\
\hline & Prehipertensión & & 72 & 72 & 72 & 73 & 74 & 75 & 75 \\
\hline & Normotensión & & 58 & 58 & 58 & 59 & 60 & 61 & 61 \\
\hline \multirow[t]{5}{*}{10} & & $\mathrm{cms}$ & 127,5 & 129,8 & 133,7 & 138,2 & 142,8 & 147,0 & 149,6 \\
\hline & Estadio 2 HTA & & 89 & 89 & 90 & 91 & 91 & 92 & 93 \\
\hline & Estadio1 HTA & & 77 & 77 & 77 & 78 & 79 & 80 & 80 \\
\hline & Prehipertensión & & 73 & 73 & 73 & 74 & 75 & 76 & 76 \\
\hline & Normotensión & & 59 & 59 & 59 & 60 & 61 & 62 & 62 \\
\hline \multirow[t]{5}{*}{11} & & $\mathrm{cms}$ & 132,4 & 135,0 & 139,4 & 144,3 & 149,2 & 15,7 & 156,4 \\
\hline & Estadio 2 HTA & & 90 & 90 & 91 & 92 & 92 & 93 & 94 \\
\hline & Estadio1 HTA & & 78 & 78 & 78 & 79 & 80 & 81 & 81 \\
\hline & Prehipertensión & & 74 & 74 & 74 & 75 & 76 & 77 & 77 \\
\hline & Normotensión & & 60 & 60 & 60 & 61 & 62 & 63 & 63 \\
\hline
\end{tabular}

Normotensión = percentil 50; Pre-Hipertensión = percentil 90 hasta $80 \mathrm{mmHg}$; Estadio 1 HTA = percentil 95; Estadio 2 HTA = percentil $99+5 \mathrm{mmHg}$. 
Tabla 2a. Mujeres. PAD (mmHg) (continuación)

\begin{tabular}{|c|c|c|c|c|c|c|c|c|c|}
\hline $\begin{array}{l}\text { Edad } \\
\text { (años) }\end{array}$ & $\begin{array}{l}\text { Percentil de Talla } \\
\text { (NHANES 2000) }\end{array}$ & & 5 & 10 & 25 & 50 & 75 & 90 & 95 \\
\hline \multirow[t]{5}{*}{12} & & $\mathrm{cms}$ & 139,2 & 142,0 & 146,5 & 151,5 & 156,4 & 160,8 & 163,5 \\
\hline & Estadio 2 HTA & & 91 & 91 & 92 & 93 & 93 & 94 & 95 \\
\hline & Estadio1 HTA & & 79 & 79 & 79 & 80 & 81 & 82 & 82 \\
\hline & Prehipertensión & & 75 & 75 & 75 & 76 & 77 & 78 & 78 \\
\hline & Normotensión & & 61 & 61 & 61 & 62 & 63 & 64 & 64 \\
\hline \multirow[t]{5}{*}{13} & & $\mathrm{cms}$ & 145,9 & 148,4 & 152,7 & 157,3 & 162,0 & 166,1 & 168,6 \\
\hline & Estadio 2 HTA & & 92 & 92 & 93 & 94 & 94 & 95 & 96 \\
\hline & Estadio1 HTA & & 80 & 80 & 80 & 81 & 82 & 83 & 83 \\
\hline & Prehipertensión & & 76 & 76 & 76 & 77 & 78 & 79 & 79 \\
\hline & Normotensión & & 62 & 62 & 62 & 63 & 64 & 65 & 65 \\
\hline \multirow[t]{5}{*}{14} & & $\mathrm{cms}$ & 149,7 & 152,1 & 156,0 & 160,5 & 164,9 & 168,9 & 171,3 \\
\hline & Estadio 2 HTA & & 93 & 93 & 94 & 95 & 95 & 96 & 97 \\
\hline & Estadio1 HTA & & 81 & 81 & 81 & 82 & 83 & 84 & 84 \\
\hline & Prehipertensión & & 77 & 77 & 77 & 78 & 79 & 80 & 80 \\
\hline & Normotensión & & 63 & 63 & 63 & 64 & 65 & 66 & 66 \\
\hline \multirow[t]{5}{*}{15} & & $\mathrm{cms}$ & 151,3 & 153,6 & 157,5 & 161,9 & 166,3 & 170,2 & 172,6 \\
\hline & Estadio 2 HTA & & 94 & 94 & 95 & 96 & 96 & 97 & 98 \\
\hline & Estadio1 HTA & & 82 & 82 & 82 & 83 & 84 & 85 & 85 \\
\hline & Prehipertensión & & 78 & 78 & 78 & 79 & 80 & 80 & 80 \\
\hline & Normotensión & & 64 & 64 & 64 & 65 & 66 & 67 & 67 \\
\hline \multirow[t]{5}{*}{16} & & $\mathrm{cms}$ & 151,9 & 154,3 & 158,2 & 162,6 & 166,9 & 170,9 & 173,2 \\
\hline & Estadio 2 HTA & & 95 & 95 & 95 & 96 & 97 & 98 & 98 \\
\hline & Estadio1 HTA & & 82 & 82 & 83 & 84 & 85 & 85 & 86 \\
\hline & Prehipertensión & & 78 & 78 & 79 & 80 & 80 & 80 & 80 \\
\hline & Normotensión & & 64 & 64 & 65 & 66 & 66 & 67 & 68 \\
\hline \multirow[t]{5}{*}{17} & & $\mathrm{cms}$ & 152,3 & 154,6 & 158,6 & 162,9 & 167,3 & 171,2 & 173,6 \\
\hline & Estadio 2 HTA & & 95 & 95 & 96 & 96 & 97 & 98 & 98 \\
\hline & Estadio1 HTA & & 82 & 83 & 83 & 84 & 85 & 85 & 86 \\
\hline & Prehipertensión & & 78 & 79 & 79 & 80 & 80 & 80 & 80 \\
\hline & Normotensión & & 64 & 65 & 65 & 66 & 67 & 67 & 68 \\
\hline
\end{tabular}

Normotensión = percentil 50; Pre-Hipertensión = percentil 90 hasta $80 \mathrm{mmHg}$; Estadio 1 HTA = percentil 95; Estadio 2 HTA = percentil $99+5 \mathrm{mmHg}$.

lina medidos en ayuno en escolares y adolescente. La insulina produce HTA por dos mecanismos: elevación de la absorción de sodio por el túbulo distal y por estimulación de la secreción de catecolaminas, debido a la hipoglicemia. Se han detectado niveles elevados de insulina en escolares y adolescentes obesos asociados a sensibilidad al sodio y aumento de la reactividad vascular, ambos efectos reversibles con la reducción del peso ${ }^{13}$.

Actualmente, hay demostraciones que insinúan que existen circunstancias en etapas de la vida intrauterina que se asocian a la hipertensión arterial y otras enfermedades en la vida adulta. De allí ha surgido el término "programación fetal", que se refiere al proceso mediante el cual un estímulo o una injuria en el período de desarrollo de un órgano o tejido tiene repercusiones posteriores permanentes ${ }^{14-15}$.

Se ha demostrado que la PA diastólica en la primera década de la vida se correlaciona inversamente con el peso de nacimiento y que esta relación aumenta con la edad ${ }^{16}$. Esta tendencia de los niños de bajo peso de nacimiento a hacer HTA estaría asociada a resistencia a la insulina, hiperlipidemia y la presencia de catecolaminas que estimularían la angiotensina II. Los individuos que nacen con bajo peso tienen ocho veces más alterado el test de tolerancia a la glucosa que los recién nacidos con peso normal. Por otra parte, la alteración del metabolismo glucosa-insulina reduce la tasa de creci- 
Tabla 2b. Hombres. PAD (mmHg)

\begin{tabular}{|c|c|c|c|c|c|c|c|c|c|}
\hline $\begin{array}{l}\text { Edad } \\
\text { (años) }\end{array}$ & $\begin{array}{l}\text { Percentil de Talla } \\
\text { (NHANES 2000) }\end{array}$ & & 5 & 10 & 25 & 50 & 75 & 90 & 95 \\
\hline \multirow[t]{5}{*}{2} & & $\mathrm{cms}$ & 81,1 & 82,4 & 84,5 & 86,9 & 89,2 & 91,4 & 92,6 \\
\hline & Estadio 2 HTA & & 71 & 72 & 73 & 74 & 75 & 76 & 76 \\
\hline & Estadio1 HTA & & 59 & 59 & 60 & 61 & 62 & 63 & 63 \\
\hline & Prehipertensión & & 54 & 55 & 56 & 57 & 58 & 58 & 59 \\
\hline & Normotensión & & 39 & 40 & 41 & 42 & 43 & 44 & 44 \\
\hline \multirow[t]{5}{*}{3} & & $\mathrm{cms}$ & 89,2 & 90,5 & 92,7 & 95,3 & 97,9 & 100,4 & 101,9 \\
\hline & Estadio 2 HTA & & 76 & 76 & 77 & 78 & 79 & 80 & 80 \\
\hline & Estadio1 HTA & & 63 & 63 & 64 & 65 & 66 & 67 & 67 \\
\hline & Prehipertensión & & 59 & 59 & 60 & 61 & 62 & 63 & 63 \\
\hline & Normotensión & & 44 & 44 & 45 & 46 & 47 & 48 & 48 \\
\hline \multirow[t]{5}{*}{4} & & $\mathrm{cms}$ & 95,6 & 97,1 & 99,7 & 102,5 & 105,4 & 108,0 & 109,5 \\
\hline & Estadio 2 HTA & & 79 & 80 & 81 & 82 & 83 & 83 & 84 \\
\hline & Estadio1 HTA & & 66 & 67 & 68 & 69 & 70 & 71 & 71 \\
\hline & Prehipertensión & & 62 & 63 & 64 & 65 & 66 & 66 & 67 \\
\hline & Normotensión & & 47 & 48 & 49 & 50 & 51 & 51 & 52 \\
\hline \multirow[t]{5}{*}{5} & & $\mathrm{cms}$ & 101,5 & 103,2 & 106,0 & 109,2 & 112,3 & 115,1 & 116,8 \\
\hline & Estadio 2 HTA & & 82 & 83 & 84 & 85 & 86 & 86 & 87 \\
\hline & Estadio1 HTA & & 69 & 70 & 71 & 72 & 73 & 74 & 74 \\
\hline & Prehipertensión & & 65 & 66 & 67 & 68 & 69 & 69 & 70 \\
\hline & Normotensión & & 50 & 51 & 52 & 53 & 54 & 55 & 55 \\
\hline \multirow[t]{5}{*}{6} & & $\mathrm{cms}$ & 107,3 & 109,2 & 112,2 & 115,7 & 119,1 & 122,1 & 123,9 \\
\hline & Estadio 2 HTA & & 85 & 85 & 86 & 87 & 88 & 89 & 89 \\
\hline & Estadio1 HTA & & 72 & 72 & 73 & 74 & 75 & 76 & 76 \\
\hline & Prehipertensión & & 68 & 68 & 69 & 70 & 71 & 72 & 72 \\
\hline & Normotensión & & 53 & 53 & 54 & 55 & 56 & 57 & 57 \\
\hline \multirow[t]{5}{*}{7} & & $\mathrm{cms}$ & 113,2 & 115,1 & 118,4 & 122,0 & 125,7 & 129,0 & 131,0 \\
\hline & Estadio 2 HTA & & 87 & 87 & 88 & 89 & 90 & 91 & 91 \\
\hline & Estadio1 HTA & & 74 & 74 & 75 & 76 & 77 & 78 & 78 \\
\hline & Prehipertensión & & 70 & 70 & 71 & 72 & 73 & 74 & 74 \\
\hline & Normotensión & & 55 & 55 & 56 & 57 & 58 & 59 & 59 \\
\hline \multirow[t]{5}{*}{8} & & $\mathrm{cms}$ & 118,8 & 120,8 & 124,3 & 128,1 & 132,1 & 135,7 & 137,8 \\
\hline & Estadio 2 HTA & & 88 & 89 & 90 & 91 & 92 & 92 & 93 \\
\hline & Estadio1 HTA & & 75 & 76 & 77 & 78 & 79 & 79 & 80 \\
\hline & Prehipertensión & & 71 & 72 & 72 & 73 & 74 & 75 & 76 \\
\hline & Normotensión & & 56 & 57 & 58 & 59 & 60 & 60 & 61 \\
\hline \multirow[t]{5}{*}{9} & & $\mathrm{cms}$ & 123,8 & 126,0 & 129,6 & 133,7 & 137,9 & 141,8 & 144,1 \\
\hline & Estadio 2 HTA & & 89 & 90 & 91 & 92 & 93 & 93 & 94 \\
\hline & Estadio1 HTA & & 76 & 77 & 78 & 79 & 80 & 81 & 81 \\
\hline & Prehipertensión & & 72 & 73 & 74 & 75 & 76 & 76 & 77 \\
\hline & Normotensión & & 57 & 58 & 59 & 60 & 61 & 61 & 62 \\
\hline \multirow[t]{5}{*}{10} & & $\mathrm{cms}$ & 128,2 & 130,5 & 134,4 & 138,8 & 143,3 & 147,4 & 149,9 \\
\hline & Estadio 2 HTA & & 90 & 91 & 91 & 93 & 93 & 94 & 95 \\
\hline & Estadio1 HTA & & 77 & 78 & 79 & 80 & 81 & 81 & 82 \\
\hline & Prehipertensión & & 73 & 73 & 74 & 75 & 76 & 77 & 78 \\
\hline & Normotensión & & 58 & 59 & 60 & 61 & 61 & 62 & 63 \\
\hline \multirow[t]{5}{*}{11} & & $\mathrm{cms}$ & 132,4 & 134,9 & 139,0 & 143,7 & 148,5 & 152,9 & 155,5 \\
\hline & Estadio 2 HTA & & 91 & 91 & 92 & 93 & 94 & 95 & 95 \\
\hline & Estadio1 HTA & & 78 & 78 & 79 & 80 & 81 & 82 & 82 \\
\hline & Prehipertensión & & 74 & 74 & 75 & 76 & 77 & 78 & 78 \\
\hline & Normotensión & & 59 & 59 & 60 & 61 & 62 & 63 & 63 \\
\hline
\end{tabular}

Normotensión = percentil 50; Pre-Hipertensión = percentil 90 hasta $80 \mathrm{mmHg}$; Estadio 1 HTA = percentil 95; Estadio 2 HTA = percentil $99+5 \mathrm{mmHg}$. 
Tabla 2b. Hombres. PAD (mmHg) (continuación)

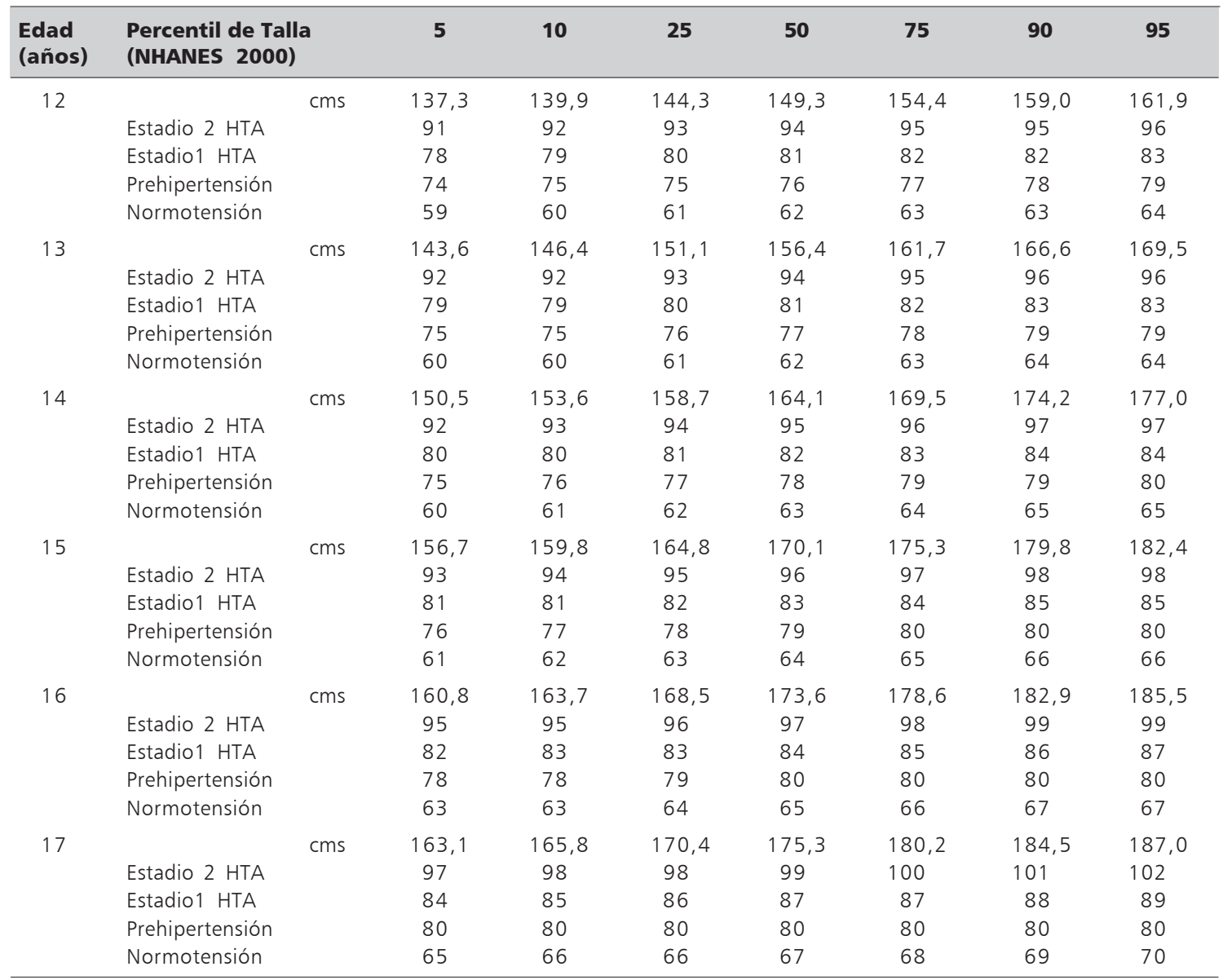

Normotensión = percentil 50; Pre-Hipertensión = percentil 90 hasta $80 \mathrm{mmHg}$; Estadio 1 HTA = percentil 95; Estadio 2 HTA = percentil $99+5 \mathrm{mmHg}$.

miento fetal, lo que se traduce en bajo peso de nacimiento. La relación HTA-bajo peso de nacimiento es mayor si además hay aumento del tamaño de la placenta. La asociación entre placenta aumentada de tamaño y déficit de peso del recién nacido sugiere desnutrición materna, luego reducir la tasa de HTA de una población debería comenzar por mejorar la nutrición de embarazadas y niños, lo que disminuiría la discordancia entre el peso de nacimiento y el tamaño placentario.

Existe evidencia experimental, en animales $\mathrm{y}$ en humanos, que cambios ocurridos a temprana edad en la carga hemodinámica pueden alterar la estructura y distensibilidad de las grandes arterias. Por otra parte, el peso de nacimien- to se relaciona inversamente con la masa del ventrículo izquierdo adulto. El mecanismo que lleva a estos cambios involucra la hormona de crecimiento, el factor de crecimiento insulinosímil tipo 1 (IGF-1) y la insulina, que actúan en el desarrollo de la pared vascular provocando aumento de la resistencia y por ende, HTA ${ }^{17-19}$.

Las investigaciones sobre el medio ambiente que favorecen la HTA en el adulto se han radicado fuertemente en la obesidad y el consumo de sal. Existe una clara asociación entre obesidad e HTA. Al relacionar el Índice de Masa Corporal (IMC) con la PA, la prevalencia de HTA se incrementa en paralelo al aumento del IMC, especialmente la HTA esencial. La obesidad y la HTA son componentes 
del Síndrome Metabólico que involucra un riesgo de enfermedad cardiovascular ${ }^{20}$.

En relación al consumo de sal un estudio cooperativo internacional de 52 Centros concluyó que la reducción de ingesta diaria de sodio

\section{Tabla 3. Causas de Hipertensión Arterial según la edad del paciente}

\footnotetext{
- Recién nacido:

- Trombosis arteria renal

- Coartación aórtica

- Estenosis arteria renal

- Malformación renal congénita (riñón poliquístico autonómico dominante y autosómico recesivo, displasia renal, hipoplasia renal unilateral, uropatías obstructivas)

- Displasia broncopulmonar

- Síndrome nefrótico congénito

- Necrosis tubular aguda

- Necrosis cortical

- Nefritis intersticial

Hiperplasia suprarrenal congénita

- Lactante y Preescolar:

- Coartación aórtica

- Enfermedad parénquima renal (uropatías obstructivas, displasia renal, nefropatía de reflujo, pielonefritis crónica, síndrome hemolítico urémico, riñón poliquístico, tumores)

- Estenosis arteria renal

- Hipercalcemia

- Feocromocitoma

\section{- Escolar:}

- Estenosis arteria renal

- Enfermedades del parénquima renal (uropatías obstructivas, displasia renal, nefropatía de reflujo, pielonefritis crónica, síndrome hemolítico urémico, riñón poliquístico, tumores, glomérulonefritis crónica, nefropatía de Schönlein Henoch)

- Hipertensión esencial

- Feocromocitoma

Estados de exceso de mineralocorticoides

\section{- Adolescente:}

- Hipertensión esencial

- Enfermedades del parénquima renal uropatías obstructivas, displasia renal, nefropatía de reflujo, pielonefritis crónica, síndrome hemolítico urémico, riñón poliquístico, tumores, glomerulonefritis crónica, nefropatía de Schönlein Henoch, lupus eritematoso diseminado)

- Estenosis de arteria renal, arteritis de Takayasu

- Hipertiroidismo

- Neurofibromatosis

- Feocromocitoma, tumores neurogénicos

Estados de exceso de mineralocorticoides
}

de $170 \mathrm{mmol}$ a $70 \mathrm{mmol}$, producía un descenso de $2 \mathrm{mmhg}$ en la PA sistólica. Esto parece insignificante, pero si tomamos la población con aumento de tamaño placentario y bajo peso de nacimiento y la sometemos a la misma baja de consumo de sodio, el rango de descenso de la PA sistólica es sobre $25 \mathrm{mmhg}$. Por otra parte si consideramos que una baja de $10 \mathrm{mmhg}$ en la PA adulta se asocia a un descenso de un 30\% de la mortalidad por causas cardiovasculares, podremos comprender que la moderación en el consumo de sal debe comenzar lo más precozmente posible ${ }^{21}$.

El pediatra debe manejar esta información para influir, tanto a nivel individual, como en el medio familiar, aplicando medidas preventivas de la HTA en todos los niños que presenten factores de riesgo.

\section{Clínica}

Los niños con PA elevada, en su gran mayoría, evolucionan asintomáticos por largos períodos y cuando presentan manifestaciones clínicas éstas suelen ser escasas e inespecíficas: cefaleas, vómitos, tinitus, epistaxis, taquicardia y en caso de HTA secundaria, sintomatología de la enfermedad causal ${ }^{22}$.

En el examen físico son importantes el peso y la estatura, pues si están alterados pueden indicar, entre otras afecciones, HTA severa. En la piel hay que buscar neurofibromas y estrías. Se debe tomar el pulso y medir la PA en las cuatro extremidades, buscar soplos en corazón, cuello, dorso y abdomen. En este último es importante la palpación de masas. El examen de fondo de ojo es muy útil para evaluar la severidad de la HTA, aunque generalmente no está alterado en los niños.

\section{Estudio}

Debe estar orientado de acuerdo a la edad del paciente, los antecedentes de la historia, hallazgos del examen físico y cifras de PA encontradas. La posibilidad de una causa subyacente debe ser considerada en cada paciente y los diagnósticos a tener en cuenta dependen de la frecuencia y edad de presentación. La evaluación incluye investigación etiológica y 
compromiso de órgano blanco ${ }^{23}$ y se distinguen dos fases ${ }^{4,6,7}$. La primera comprende un hemograma para evaluar la existencia de anemia, propia de estados avanzados de insuficiencia renal e intoxicación por metales pesados, y trombocitopenia en casos de síndrome hemolítico urémico; estudio de proteinuria ó hematuria, hallazgos usuales en casos de glomerulopatías, litiasis, tumores, o enfermedad renal quística; examen de orina completa y urocultivo para descartar infección del tracto urinario, y glucosuria ante la sospecha de diabetes mellitus o tubulopatías. Igualmente medición de creatinina y nitrógeno ureico en plasma para evaluar la velocidad de filtración glomerular, gases y electrolitos en plasma y orina para diagnosticar enfermedades de la excresión de potasio, como un hiperaldosteronismo en los casos de hipokalemia. La presencia de alcalosis metabólica orientará esta sospecha diagnóstica. En la evaluación de la hipokalemia debe tenerse en cuenta el uso de fármacos que reducen los niveles de potasio (diuréticos, laxantes, corticoides). Los fármacos que bloquean el sistema reninaangiotensina (SRA) pueden aumentar los niveles de potasio y enmascarar la hipokalemia. Perfil lipídico, curva de tolerancia a la glucosa y curva de insulina, especialmente en el paciente obeso, o con historia familiar. La ecografía renal permite el diagnóstico de malformaciones urológicas, urolitiasis, tumores, enfermedad renal quística y daño renal crónico.

Otros estudios son una polisomnografía en caso de trastornos del sueño, ecocardiograma y EGG, buscando hipertrofia ventricular izquierda (HVI), que es el hallazgo más relevante del compromiso de órganos blancos. Fondo de ojo: cuando aparecen alteraciones de los vasos de la retina orientan a la cronicidad o a cifras de PA elevadas. T3, T4 y TSH, para descartar hipertiroidismo.

Si no se llega a conclusiones definitivas en cuanto a la etiología de la HTA con los exámenes de rutina se deben realizar otros, de acuerdo a la anamnesis y hallazgos en el examen físico y el estudio de laboratorio inicial. En esta segunda fase puede usarse: ecografía doppler renal, uretrocistografía, cintigrama renal con captopril, renina plasmática, catecolaminas urinarias,esteroides plasmáticos y urinarios, arterio- grafía clásica, angiografía por sustracción digital, angioresonancia nuclear magnética, tomografía computada en 3D,cintigrama renal con metaiodobenzilguanidina (MIBG) y eventualmente biopsia renal.

\section{Estudio complementario}

La monitorización ambulatoria de presión arterial (MAPA) es un procedimiento cada vez más utilizado en la población pediátrica dado su gran utilidad. A través de la instalación de un monitor durante las 24 horas del día se registran múltiples tomas de PA. Es un procedimiento bien tolerado. Sus ventajas las da el contar con un gran número de mediciones (cada $20 \mathrm{~min}$ durante el día y cada 30 min durante el período nocturno), realizadas durante la actividad regular del niño en un ambiente habitual para él que evita el efecto de delantal blanco, y todo esto sumado a que este tipo de monitorización permite medición y evaluación de otros parámetros concomitantes como lo son la variabilidad circadiana (día/noche) y la variabilidad intrínseca (desviación estándar) ${ }^{5,24,25}$.

La MAPA es de gran utilidad en la evaluación de HTA del delantal blanco, HTA enmascarada, daño a órgano blanco, aparente resistencia a drogas, hipotensión inducida por drogas y en la suspensión del tratamiento hipotensor. Da información adicional de la PA en HTA episódica, enfermedad renal crónica, Diabetes Mellitus, disfunción autonómica, y en el diagnóstico de HTA enmascarada en población en riesgo (adolescente, obeso, recién nacido con bajo peso de nacimiento, niño con antecedentes familiares de HTA, Diabetes Mellitus, etc). La HTA enmascarada es aquella en que el niño es normotenso en la consulta clínica, pero resulta hipertenso con la MAPA. Se ha descrito que el tener HTA enmascarada es un precursor de HTA sostenida e HVI.

La MAPA debe ser realizada por médicos con experiencia en su uso e interpretación. Los valores deben ser comparados con estudios poblacionales de monitoreo de 24 horas y no con las tablas de toma de presión casual. Actualmente, se considera a la MAPA como un método simple de realizar en niños, útil en el diagnóstico precoz de algunas patologías y que permite identificar la HTA con mayor certeza. 


\section{Tratamiento}

La prevención de la HTA en los pacientes con factores de riesgo conocido se logra con un grupo de medidas que se conocen como "cambios de estilo de vida" que incluyen: restricción de las sales de sodio, aumento de la ingesta de potasio, mantención de ingesta baja en grasas saturadas, disminución del peso, estimulación de actividad física regular, evitar las situaciones de estrés, consumo bajo o eliminación del alcohol, evitar el cigarrillo y el uso de medicamentos como antigripales, anticonceptivos, corticoides y aines por ser hipertensores ${ }^{7,26,27}$.

En cuanto a la alimentación, la HTA se relaciona con la obesidad, especialmente en los adolescentes. La reducción de peso y la realización de ejercicios físicos aeróbicos, al menos tres veces por semana, disminuyen la concentración de triglicéridos y aumenta la fracción de HDL, disminuyendo así el factor de riesgo de la hiperlipidemia. La exposición a dietas altas en sodio en la niñez llevan a cifras más elevadas de PA y éstas persisten aunque después se elimine la sal de la dieta. En niños y adultos se han obtenido bajas significativas de la PA con altas ingestas de potasio. Hay una leve relación inversa entre el calcio de la dieta y la aparición de HTA. Lo mismo ocurriría con el magnesio.

El niño con factores de riesgo de HTA debe considerarse como marcador de una predisposición que puede afectar a toda la familia. Es importante involucrar a todo el núcleo familiar en los cambios de estilos de vida, lo que facilita la adherencia del niño a ellas y previene o retarda la HTA en otros hermanos predispuestos.

\section{Tratamiento farmacológico}

El manejo de la HTA en pediatría comprende la corrección de las causas secundarias si es que las hubiese, realizar refuerzo en relación a cambios en estilos de vida y evaluar la necesidad de tratamiento farmacológico ${ }^{28}$.

La mayoría de los niños que presentan HTA, tarde o temprano requieren de tratamiento farmacológico, aunque existen reportes de pacientes que con sólo la modificación dietética y de hábitos lo han logrado. La HTA secundaria en general es más difícil de tratar que la esencial en cuanto a su control y prevención de efectos secundarios, requiriéndose con frecuencia tratamientos multiasociados para lograr un resultado satisfactorio.

Los fármacos se indican cuando la modificación de los estilos de vida no ha logrado el resultado esperado, y en situaciones especiales que se detallan más adelante. El tratamiento debe ser individualizado, dependiendo del nivel de PA y el grado de respuesta al tratamiento no farmacológico iniciado ${ }^{29}$. Siempre se debe tener presente que:

1. El objetivo debe ser la reducción de las cifras a niveles bajo el percentil 95 para edad, sexo y talla en pacientes sin complicaciones y bajo el percentil 90 si ya hay compromiso en órganos blancos.

2. El cumplimiento del tratamiento es inversamente proporcional a su complejidad, el número de drogas usadas y su fraccionamiento en el día.

3. En general debe ser iniciado con una droga y cuando se deban usar más de una, la elección debe considerar que actúen en sistemas fisiológicos diferentes.

4. Las drogas de mayor uso en niños en orden de frecuencia son bloqueadores de la enzima de conversión de angiotensina (IECAS), bloqueadores de receptores de angiotensina II, beta bloqueador, antagonista alfa y beta adrenérgicos, bloqueadores de canales del calcio, vasodilatadores y diuréticos.

Desde 1998, la Food and Drug Administration (FDA) ha obligado a los laboratorios farmacológicos a informar sobre dosis pediátricas. Esto ha derivado en la realización de investigaciones, que permiten disponer de información sobre la seguridad y eficacia de las drogas antihipertensivas en uso en niños, lo que nos permite terapias más racionales.

A partir de 1977, se han publicado consensos sobre HTA pediátrica que han servido en la evaluación y el tratamiento de estos pacientes. La más reciente de ellos es la Fourth Report on the Diagnosis Evaluation and Treatment of High Blood Pressure in Children publicado en el 2004 que provee recomendaciones para el tratamiento de la HTA en niños en base a la mejor evidencia disponible $e^{7}$. 
$\mathrm{Al}$ iniciarse el tratamiento debe tomarse en consideración la severidad de la HTA, la presencia de HTA secundaria, el compromiso de órganos blancos como la HVI y problemas agregados como obesidad, diabetes mellitus y enfermedad renal. Estos aspectos están incorporados en el algoritmo de tratamiento (figura 1).

Las indicaciones de iniciar tratamiento antihipertensivo son: HTA en etapa 2, HTA secundaria, HTA sintomática, HTA con daño de órgano blanco, Diabetes Mellitus (tipo 1 y 2) e HTA persistente que no responde a medidas no farmacológicas (6 meses).

Actualmente, se posee un arsenal terapéutico (tabla 4) con gran variedad de mecanismos de acción, como son: 2,7,27-29

Diuréticos de diversos mecanismos de acción: tiazidas (Hidroclorotiazidas), diuréticos de asa (Furosemida), ahorradores de potasio (Espironolactona, Amiloride). Actúan disminuyendo la concentración de sodio en el músculo liso vascular, con la consiguiente disminución de la reactividad ante estímulos presores.

Betabloqueadores (Atenolol, Bisoprolol, Propanolol), cuyo mecanismo de acción no es bien conocido pero reducen la PA y el gasto cardíaco.

Alfabloqueadores: agonistas 1-2 y antagonistas 1 (Doxasosina, Prazosin, Clonidina, Terazosina), disminuyen la resistencia periférica.
Acción central (Alfametildopa, Clonidina), disminuyen la actividad adrenérgica.

Fármacos de acción múltiple (Labetalol), combinan en la misma molécula bloqueo alfa y betaadrenérgico.

Vasodilatadores (Hidralazina, Minoxidil, Nitroprusiato), actúan sobre la fibra lisa muscular de los vasos de resistencia al incrementar las concentraciones de GMP cíclico.

Inhibidores de la enzima de conversión de la angiotensina (IECA) (Captopril, Enalapril, Lisinopril, Ramipril), actúan reduciendo la generación de angiotensina II tanto circulante como en pared vascular e incrementando la concentración de bradicininas.

Bloqueadores de receptores de angiotensina. Este grupo se caracteriza por el bloqueo selectivo de los receptores AT1 de angiotensina II (ARA II) (Losartán, Ibersartán, Candesartan). Estos y los IECA comparten algunos de los mecanismos de acción antihipertensiva, como es la disminución de la actividad de la angiotensina II.

Antagonistas de calcio (Nifedipino, Amlodipino), disminuyen la contractibilidad de la fibra muscular lisa al disminuir las concentraciones intracelulares de calcio.

La terapia farmacológica debe ser monitorizada cada 2 a 4 semanas inicialmente para comprobar su eficacia y los potenciales efectos adversos. Una vez alcanzado un control ade-

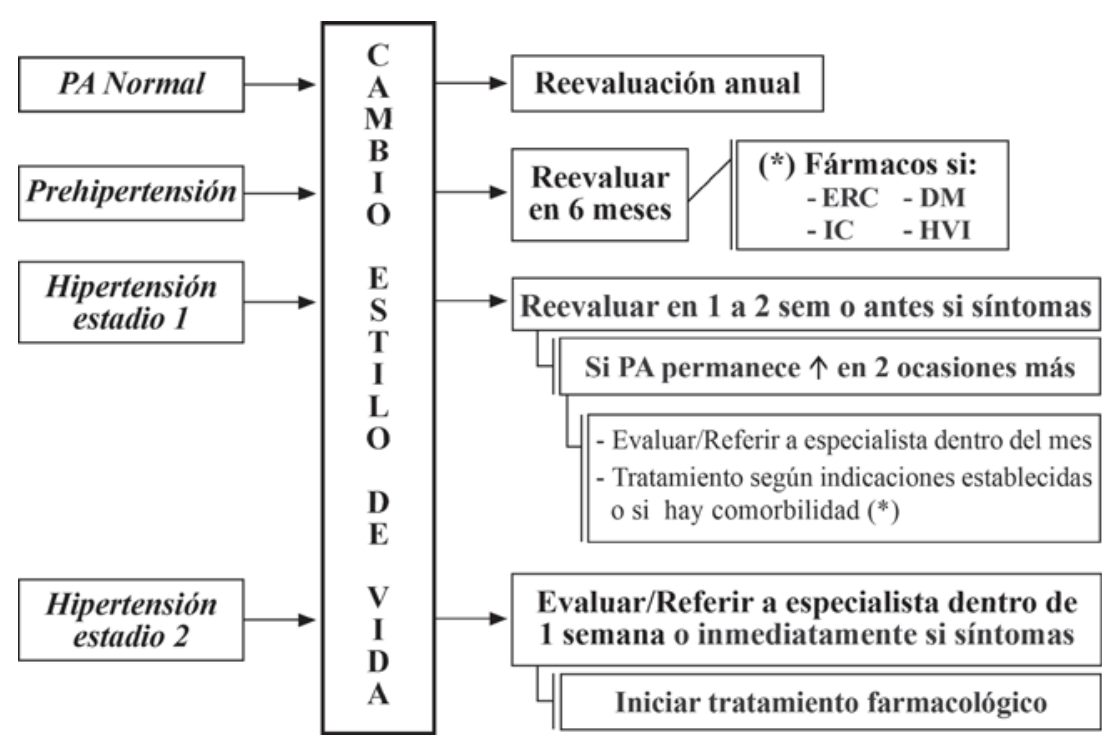

Figura 1. Algoritmo de tratamiento de la Hipertensión Arterial. *ERC $=$ Enfermedad Renal Crónica, $\mathrm{DM}=$ Diabetes Mellitus, IC = Insuficiencia Cardíaca, HVI= Hipertrofia Ventrículo Izquierdo. 
Tabla 4. Medicamentos antihipertensivos de uso en pediatría

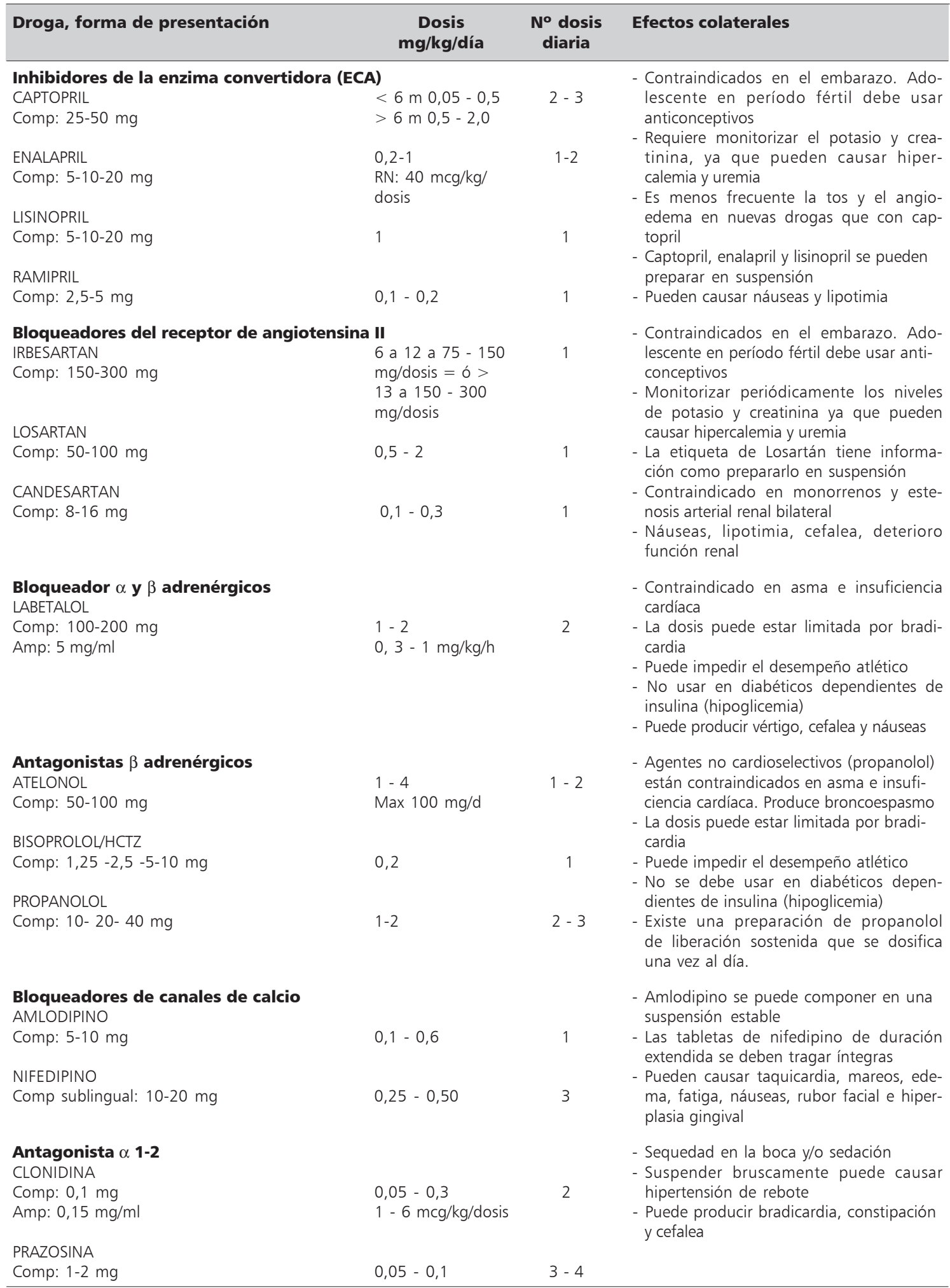


Tabla 4. Medicamentos antihipertensivos de uso en pediatría (continuación)

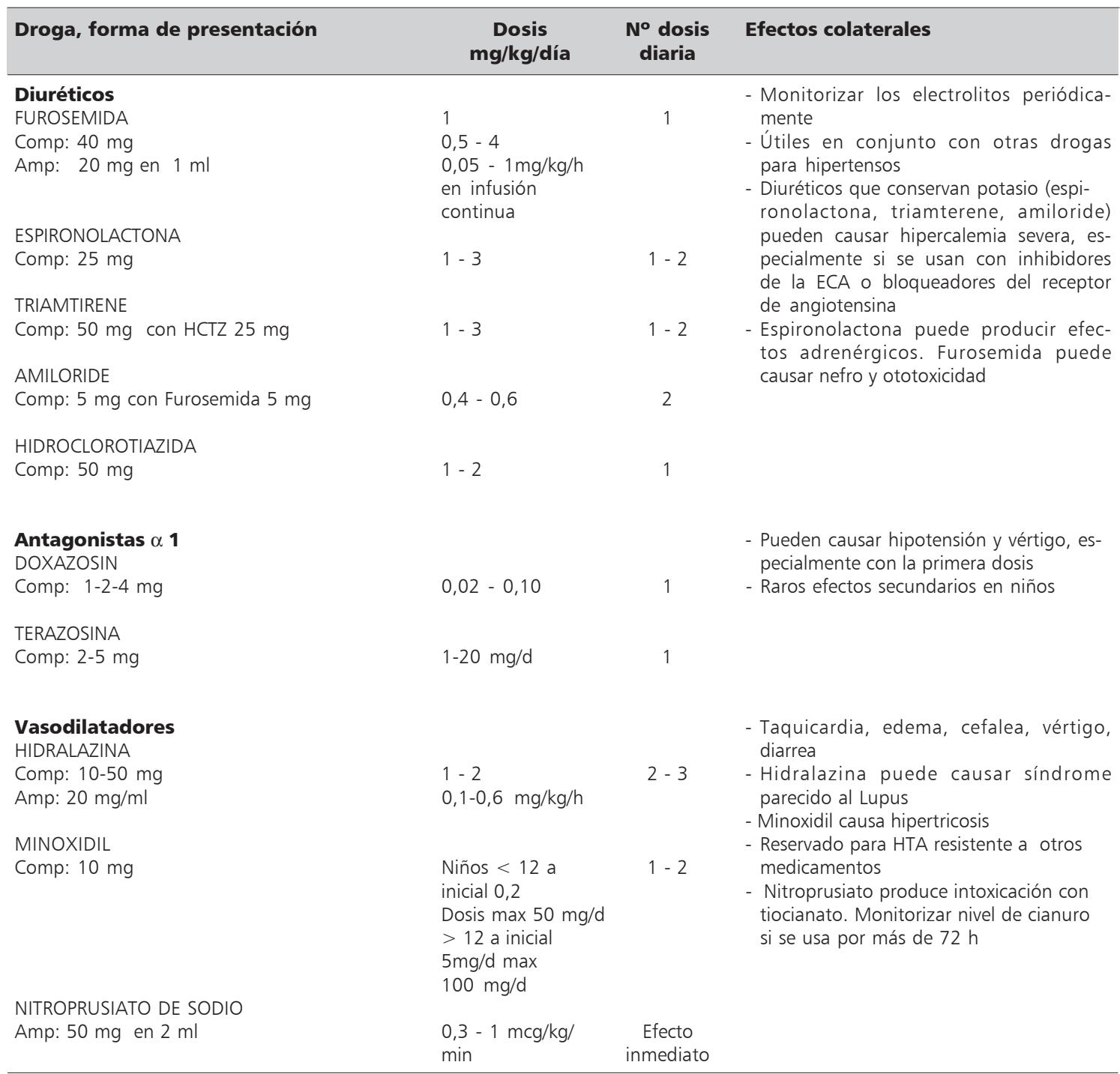

cuado de la PA, ésta debe medirse cada 3 a 4 meses junto a exámenes de laboratorio, en especial, si se usan agentes que afecten el sistema renina angiotensina o diuréticos ${ }^{29}$.

\section{Vacunas}

En etapa experimental en ratas y algunos humanos está la modificación del sistema renina angiotensina provocando cambios inmunológicos mediante el uso de vacunas para controlar el alza de la $\mathrm{PA}^{32-34}$.

\section{Conclusiones}

La hipertensión arterial en pediatría ha tomado cada vez mayor importancia, y a pesar que su prevalencia es relativamente baja es una patología que debe ser tomada en cuenta dado que su inicio se ha establecido en etapas tempranas de la vida, incluso en algunos casos en la vida intrauterina. El daño cardiovascular y a otros parénquimas producto de la HTA ha hecho plantear cada vez más la importancia de la 
toma rutinaria de la presión arterial, y el monitoreo ambulatorio depresión arterial de 24 horas es un procedimiento cada vez más utilizado en la población pediátrica dada su gran utilidad para el diagnóstico y pronóstico de la enfermedad. El tratamiento siempre debe ser iniciado con medidas no farmacológicas o mejor llamadas estilos de vida saludables, que han sido detalladas en las secciones precedentes, y el tratamiento farmacológico será indicado cuando la modificación de los estilos de vida no ha logrado el resultado esperado. El pediatra debe ser un participante activo en la búsqueda de la HTA, dado las múltiples instancias en que es posible realizar una labor preventiva,en especial debido al notorio aumento de la HTA primaria que se ha observado en pediatría en los últimos años.

\section{Referencias}

1.- Norwood VF: Hypertension. Pediatr Rev 2002; 23: 197-208.

2.- Lurbe E, Torró I, Cremades B: Hipertensión arterial en niños y adolescentes. Protocolos, diagnósticos y terapéuticos. En Malaga S (ed). Nefrourología Pediátrica Madrid 2005; 155-64.

3.- Lagomarsino E: Rol del pediatra en la prevención de la hipertensión esencial del adulto. Rev Méd Chile 1999; 127: 349-57.

4.- Lagomarsino E, González E, Solar E, Cavagnaro F, Saieh C: Hipertensión arterial en pediatría. Pautas de nefrología infantil. Rev Chil Pediatr 1997; 1: $27-$ 42.

5.- Hadtstein Ch, Schaefer F: What adult nephrologist should know about childhood blood pressure. Nephrol Dial Transplant 2007; 22: 2119-23.

6.- Saieh C: Hipertensión arterial. En: Saieh C., Izzo C. J.M. Escala (Ed). Manual de Nefrourología 3ª ed. 2001. Publicaciones Mediterráneo, Santiago, Chile. Pág: 1415.

7.- The fourth report on the diagnosis, evaluation and treatment of high blood pressure in children and adolescents. Pediatrics 2004; 114: 555-76.

8.- Gilman M, Cook N, Rosner B, Evans D, Keough M, Taylor J, Hebert P, Hennekens Ch: Prediction of adult blood pressure from childhood values. Am J Epidemiol 1991; 134: 730-9.

9.- Lurbe E: Nuevos hitos en la evaluación de la presión arterial en niños y adolescentes. An Pediatr (Barc) 2006; 64: 111-3.

10.- Ingelfinger JR, Dillon MJ: Evaluation of secondary hypertension. En Pediatric Nephrolgy, Third Edition. Eds. Holliday M, Barrat TM, Avner ED. Williams \&
Wilkins, Baltimore, Maryland, U.S.A. 1993, pag: 1146-64.

11.- Lagomarsino E: Hipertension arterial. Horizonte actual y responsabilidad del pediatra. Rev Chil Pediatr 1998; 69: 227-35.

12.- Saieh C: Factores de riesgo en hijos de padres hipertensos. Rev Med Clínica Las Condes 1995; 6: 15-7.

13.- Van den Elzen AP, de Ridder MA, Grobbee DE, Witteman JC, Utierwaal CS: Families and the natural history of blood pressure. J Hypertens 2004; 17: 93640.

14.- Barker DJP, Bull AR, Osmond C, Simmonds SJ: Fetal and placental size and risk of hypertension in adult life. BMJ 1990; 301: 259-62.

15.- Law CM, de Swiet M, Osmond C, Fayers PM, Barker $D J P$ : Initiation of hypertension in utero and its amplification throughout life. BMJ 1993; 306: 24-7.

16.- Mcneill G, Tuya C, Smith W: The role of genetic and environmental factors in the association between birth weight and blood pressure: evidence from meta-analysis of twin studies. Int J Epidemiol 2004; 33: 995-1001.

17.- Martyn CN, Greenwald: Impaired synthesis of elastin in walls of aorta and large conduit arteries during early development as an initiating event in pathogenesis of systemic hypertension. Lancet 1997; 350: 953-55.

18.- Barker DJP, Fall CHD: Fetal and infant origins of cardiovascular disease. Arch Dis Child 1993; 68: 7979.

19.- Bergvall N, Iliadou A, Tuvemo T, Cnattingius S: Birth characteristics and risk of high systolic Blood pressure in early adulthood. Epidemiology 2005; 635-40.

20.- Rocchini AP, Match V, Anderson J, et al: Blood pressure in obese adolescents: effects of weight loss. Pediatrics 1988; 82: 16-23.

21.- Stamler J, Rose G, Elliot P, Anderson RM, May RM: Findings of the International Cooperative Inter Salt study. Hypertension 1991; 17: 1-9.

22.- Varda N, Gregoric A: A diagnostic approach for the child with hypertension. Pediatr Nephrol 2005; 20: 499-506.

23.- Portman RJ, Mcniece KL, Swinford RD, Braun MC, Samules JA: Pediatric hypertension: diagnosis, evaluation, management, and treatment for the primary care physician. Curr Probl Pediatr Adolesc Health Care 2005; 35: 257-304.

24.- Saieh C: 24 hour blood pressure monitoring in children: incidence of white coat hypertension. (Abstract) Pediatr Nephrol 2004; 19: 167.

25.- Flynn J: Differentiation between primary and secondary hypertension in children using ambulatory blood pressure monitoring. Pediatrics 2002; 110: 8993.

26.- Vollmer VM, Sacks FM, et al: Effects of diet and sodium intake on blood pressure: subgroup analysis of the DASH-sodium trial. Ann Intern Med 2001; 135: 1019-28.

27.- Díaz S, Bonilla-Félix M: Tratamiento de la hipertensión arterial en niños. Arch Latin Ped 2005; 5: 89-99. 
28.- Lagomarsino E: Tratamiento farmacológico de la hipertensión arterial en pediatría. Rev Chil Pediatr 2000; 71: 380-9.

29.- Flynn J, Daniels SR: Pharmacologic treatment of hypertension in children and adolescents. J Pediatr 2006; 149: 746-54.

30.- Nahata MC: Lack of pediatric drug formulation. Pediatrics 1999; 104: 607-9.

31.- Flynn JL: Successes and shortcomings of the Food and Drug modernization act. Am J Hipertens 2003; 16: $889-91$.
32.- Ménard J: A vaccine for hypertension. J Hypertens 2007; 25: 41-6.

33.- Ambüh PM, Tissot AC, Fulurija A, Maurer $P$, Nussberger J, Sabat $R$, et al: A vaccine for hypertension based on virus-like particles: preclinical efficacy and phase I safety and immunogenicity. J Hypertens 2007; 25: 63-72.

34.- Krum H, Gilbert R: Novel therapies blocking the rennin-angiotensin-aldosterone system in the management of hypertension and related disorders. J Hypertens 2007; 25: 25-35. 